\title{
Dryopteris x fraser-jenkinsii Gibby \& Widén en Galicia y nuevas localidades de Cystopteris diaphana (Bory) Blasdell para la provincia de A Coruña (NW de la península ibérica)
}

\author{
X. Ignacio González-Martínez ${ }^{1}$ y Carlos Boullón Agrelo² \\ ${ }^{1}$ Avda. Miguel Rodríguez Bautista, 23. 15960, Ribeira (A Coruña) \\ ${ }^{2} \mathrm{r} / 1$ da Angustia, 114. 15948, A Pobra do Caramiñal (A Coruña)
}

\section{Correspondencia}

X.I. González-Martínez

e-mail: xoseignaciogonzalez@yahoo.es

Recibido: 25 mayo 2017

Aceptado: 29 agosto 2017

Publicado on-line: diciembre 2017

\begin{abstract}
Resumen
En esta nota presentamos información sobre el híbrido interespecífico Dryopteris $\times$ fraser-jenkinsii Gibby \& Widén para el territorio de Galicia, así como un conjunto de nuevas localidades de Cystopteris diaphana (Bory) Blasdell para la provincia de A Coruña. Todos los pliegos testigo se hallan depositados en el Herbario SANT de la Universidad de Santiago de Compostela.
\end{abstract}

Palabras clave: Flora vascular, pteridófitos, corología, taxonomía, ecología, Galicia, NW ibérico.

\begin{abstract}
Dryopteris $\times$ fraser-jenkinsii Gibby \& Widén in Galicia and new localities of Cystopteris diaphana (Bory) Blasdell inACoruña province (NW Iberian Peninsula)

In this note, information of interest about the interespecific hybrid Dryopteris $\times$ fraser-jenkinsii Gibby \& Widén in Galician territory is presented. In addition, several new localities of Cystopteris diaphana (Bory) Blasdell for A Coruña province are also presented. All the cited specimens are deposited at the SANT Herbarium.
\end{abstract}

Key words: Vascular flora, pteridophyte, chorology, taxonomy, ecology, Galicia, Iberian NW.

\section{Introducción}

Fruto de diversas prospecciones realizadas en bosques de vaguada localizados en la fachada atlántica del SW de la provincia de A Coruña, damos a conocer la presencia del híbrido interespecífico Dryopteris $\times$ fraser-jenkinsii Gibby \& Widén, no documentado en firme con anterioridad en Galicia, así como un conjunto de nuevas localidades de Cystopteris diaphana (Bory) Blasdell para dicha provincia.

Para los taxones estudiados se indican los datos geográficos del lugar de recolección, las coordenadas UTM [X, Y (m), 29T; ETRS89], altitud (m s.n.m), ecología, fecha de recolección, colector/es y número identificador de herbario.
Además, se hacen diversos comentarios sobre aspectos corológicos, ecología y características morfológicas. Todos los pliegos testigo se hallan depositados en el Herbario SANT de la Universidad Santiago de Compostela.

Para conocer la relevancia de los hallazgos, se han consultado diversas floras, trabajos científicos, catálogos y otro tipo de publicaciones que figuran en la bibliografía. Además, se realizaron diversas consultas en el mencionado Herbario SANT y en el portal electrónico de datos GBIF (Global Biodiversity Information Facility, www.gbif.es). En las referencias a dicho recurso electrónico, aparece indicada la fecha de consulta del mismo en el texto.

En cuanto a la nomenclatura taxonómica, para 
el género Dryopteris hemos seguido lo expuesto en Flora iberica (Salvo \& Arrabal, 1986), mientras que para Cystopteris se han consultado otras fuentes más actualizadas, como por ejemplo los trabajos de Murphy \& Rumsey (2005) y Prelli (2015).

Finalmente, el objetivo principal de esta nota es seguir actualizando en la medida de lo posible el conocimiento sobre la pteridoflora en el ámbito de Galicia.

\section{Resultados}

\section{Dryopteris $\times$ fraser-jenkinsii Gibby \& Widén}

ESPAÑA. A Coruña. Muros, Torea, margen derecho del "Rego dos Balados" (tributario del río Rateira), 496928, 4741841, c. $203 \mathrm{~m}$, sobre un talud terroso con elevada cubierta muscinal, dominada por los briófitos Rhytidiadelphus loreus, Plagiothecium undulatum y Thuidium tamariscinum, en la ladera de umbría del valle y bajo dosel de Quercus robur, Laurus nobilis y Pyrus cordata (Rusco-Quercetum roboris), en compañía de Blechnum spicant subsp. spicant, Dyopteris affinis subsp. affinis, Hedera hibernica y Rubus gr. ulmifolius, 24-IX-2016, X. Ignacio GonzálezMartínez, Carlos Boullón Agrelo \& Antía Queiruga del Alisal (SANT 72813).

Se trata de una notoespecie originada por hibridación interespecífica entre Dryopteris affinis (Lowe) Fraser-Jenk. subsp. affinis y $D$. dilatata (Hoffm.) A. Gray o D. guanchica Gibby \& Jermy, y cuyo hallazgo en la presente localidad supone novedad para la pteridoflora gallega. Su presencia en nuestro territorio no es de extrañar, habida cuenta de la elevada frecuencia con la que se producen hibridaciones entre los miembros del género Dryopteris Adans. (Pteridophyta, Dryopteridaceae), un proceso que en estos helechos se encuentra muy a menudo ligado a fenómenos de especiación (Wagner, 1971; FraserJenkins, 1982; Fraser-Jenkins \& Reichstein, 1984; Quintanilla et al. 2006; Pérez Carro \& Fernández Areces, 2007, 2013, 2016). Como consecuencia de esta elevada tasa de hibridación, más de la mitad de los integrantes de este conflictivo género son de naturaleza alopoliploide (Quintanilla et al. op. cit.). Estos fenómenos de introgresión genética son especialmente manifiestos dentro del denominado complejo "D. dilatata" (Gibby, 1983).

El conjunto de rasgos morfológicos que nos han llevado a esta determinación taxonómica, además del evidente aspecto intermedio entre los taxones arriba indicados, y a su vez apoyados en las apreciaciones de Pérez Carro \& Fernández Areces (2007), fueron (vid. Fig. 1 \& 2): (1) la acusada desigualdad en el tamaño de las pínnulas basioscópica proximal y la acroscópica correspondiente del par de pinnas basales, ambas pinnatisectas, rasgos asociados, entre otras especies, a $D$. dilatata y $D$. guanchica (Salvo \& Arrabal, 1986; Prelli, 2015); (2) la profunda división de las últimas 2-3 pínnulas proximales, en especial hacia la mitad inferior de la lámina, unido a la presencia de pequeñas aristas apicales en los dientes de las pínnulas (Fig. 2). En D. filix-max (L.) Schott, especie que no hemos visto en la cuenca del río Rateira, dichas pínnulas no llegan a estar tan profundamente divididas y no es tan evidente la diferencia de tamaño entre ellas (Salvo \& Arrabal, 1986; Prelli, 2015). Además, la presencia de pequeñas aristas en la terminación de los segmentos de último orden suele ser un carácter ligado a especies como $D$. dilatata y $D$. guanchica, entre otros taxones, mientras que en $D$. filix-max los dientes son agudos pero sin llegar a portar aristas del tipo "dilatata" (Salvo \& Arrabal, 1986); y (3) la escasez de páleas en toda la longitud del pecíolo y el raquis, carácter este último que descartaría la

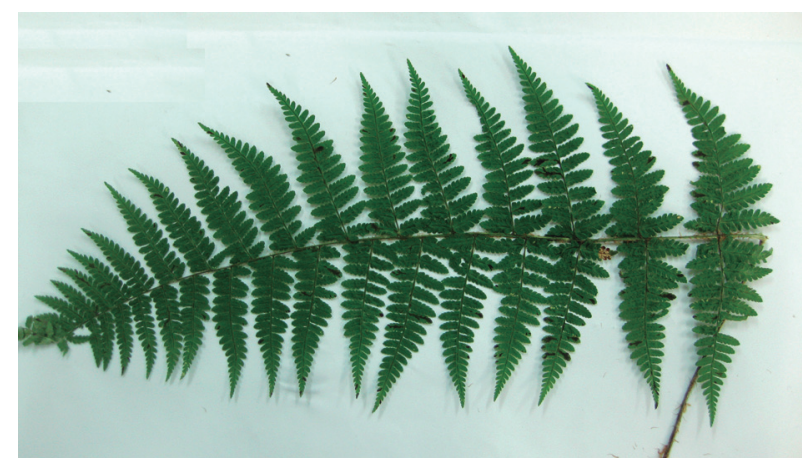

Figura 1. Fronde de Dryopteris $\times$ fraser-jenkinsii en herbario. (A Coruña). Material recolectado en Muros ( $A$ Coruña). Figure 1. Frond of Dryopteris $\times$ fraser-jenkinsii in herbarium. Material collected in Muros (A Coruña).

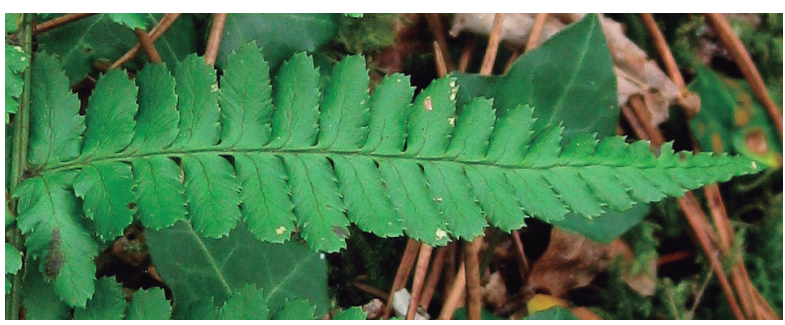

Figura 2. Detalle de una pinna de Dryopteris $\times$ fraserjenkinsii en el campo, donde puede observarse la presencia de pequeñas aristas en el ápice de los dientes de los segmentos de último orden. Muros (A Coruña); 24-IX-2016. Figure 2. Detail of a pinna of Dryopteris $x$ fraser-jenkinsii in the field, with pinnules showing aristate teeth apices (A Coruña); 24-IX-2016. 
posible confusión con alguno de los integrantes del grupo de $D$. affinis, los cuales presentan el raquis de la fronde densamente paleáceoSalvo \& Arrabal, 1986; Ivanova, 2004; Prelli, 2015). En definitiva, $D$. fraser-jenkinsii muestra un aspecto intermedio entre $D$. affinis subsp. affinis y $D$. dilatata $0 D$. guanchica: la forma general de la lámina y la apariencia de las pínnulas más distales recuerdan al primero, mientras que la forma y tamaño de las pínnulas proximales y la presencia de aristas en los dientes de los segmentos de último orden son rasgos que apuntan hacia los segundos. Finalmente, el material observado en el campo no presentaba esporangios desarrollados.

En el lugar de recolección, D. guanchica y $D$. affinis subsp. affinis fueron, con diferencia, los representantes mayoritarios del género, especialmente el primero, estando $D$. dilatata mal representado, al menos en el momento de nuestras prospecciones en el Rego dos Balados (cuenca del río Rateira). Por este motivo, creemos que $D$. guanchica y $D$. affinis subsp. affinis son los parentales más probables en la presente localidad. Además, el aspecto "espinuloso" de las pínnulas debido a la presencia de pequeñas aristas en su periferia, recuerda al que puede observarse habitualmente en $D$. guanchica (obs. pers.). En cuanto a la abundancia de este híbrido en la zona, solo hemos podido ver un par de ejemplares, los cuales se encontraban próximos entre ellos.

Finalmente, en el Herbario SANT hemos encontrado un pliego determinado como $D$. affinis s.l. con material recolectado en la localidad de Vila de Cruces (Pontevedra) (Amigo \& Louzán s.n.; SANT 41738, 10-Vl-1999), y que por sus características morfológicas también habría que llevarlo al presente híbrido. Además, en la base de datos GBIF [03-VIII-2017] hemos encontrado un pliego MA asignado a $D . \times$ fraser-jenkinsii (Alejandre \& Gil, s.n.; MA 484837, 15-10-1989), colectado en la fraga del río Mandeo (Coirós, A Coruña), el cual habría que revisar con el fin de confirmar la determinación taxonómica, en cuyo caso esta sería la primera recolección para Galicia.

\section{Cystopteris diaphana (Bory) Blasdell $\equiv$ Polypodium diaphanum Bory = Cystopteris viridula (Desv.) Desv.}

ESPAÑA. A Coruña. Lousame, Vilacoba, Comparada, 516990, 4736616, c. $290 \mathrm{~m}$, en talud terroso y muy húmedo en la ladera de umbría del valle del río Vilacoba, bajo dosel de Quercus robur y Corylus avellana (Rusco-Quercetum roboris subass. violetosum rivinianae), acompañado de Chrysosplenium oppositifolium, Saxifraga spathularis, Oxalis acetosella, Hedera hibernica, Umbilicus rupestris y Cardamine flexuosa, y con la presencia de numerosos briófitos, entre los que figuran Thamnobryum alopecurum, Fissidens serrulatus, F. polyphyllus, Plagiothecium nemorale, Isothecium alopecuroides, Kindbergia praelongum, Thuidium tamariscinum, Hookeria lucens y Pellia epiphylla, 21-II-2016, X. Ignacio González-Martínez \& Carlos Boullón Agrelo (SANT 72621); ibidem, Outes, San Lourenzo de Matasueiros, margen derecho del río San Paio, 504224, 4747566, c. $250 \mathrm{~m}$, talud terroso muy húmedo en la vertiente de umbría del valle, bajo ripisilva de Corylus avellana, y en compañía de Athyrium filix-femina, Polystichum setiferum, Hedera hibernica, Oxalis acetosella y los briófitos, Fissidens serrulatus, Thuidium tamariscinum, Isothecium alopecuroides, Plagiothecium nemorale y Pellia epiphylla, 06-III-2016, X. Ignacio González-Martínez \& Carlos Boullón Agrelo (SANT 72622); ibidem, Lousame, Vilacoba, cerca de O Inferniño, entre las localidades de Vilacoba y Vilar de Reconco, valle del "Rego da Candosa", tributario del río Vilacoba, 515002, 4735706, c. $200 \mathrm{~m}$, en grieta de bloque rocoso vertical y rezumante, bajo dosel de Quercus robur, Corylus avellana, Castanea sativa y Salix atrocinerea, en compañía de Athyrium filixfemina, Umbilicus rupestris, Cardamine flexuosa y de los briófitos Mnium hornum, Fissidens serrulatus, Diplophyllum albicans y Lyphocolea cf. heterophylla, 15-V-2016, X. Ignacio GonzálezMartínez \& Carlos Boullón Agrelo (SANT 72666); ibidem, Boiro, Cures, Covelo, margen derecho del "Rego da Devesa da Graña" (cuenca del río Coroño), 507334, 4727461, c. 295, $\mathrm{m}$ sobre rocas silíceas recubiertas de briófitos, al lado del curso de agua, en orientación $\mathrm{N}$, bajo dosel ripario de Alnus glutinosa, Laurus nobilis y Quercus robur, en compañía de Hedera hibernica y Asplenium obovatum subsp. billotii, 12-VIII-2016, X. Ignacio González-Martínez \& Carlos Boullón Agrelo (SANT 72773); ibidem, Mazaricos, Chacín, margen izquierdo del río Santa Baia (cuenca del río Entíns), 505357,4748835 , c. $90 \mathrm{~m}$, sobre talud terroso en umbría permanente y bajo ripisilva de Corylus avellana, acompañado de Athyrium filix-femina, Oxalis acetosella, Hypericum androsaemum y Geranium robertianum, así como de los briófitos Thamnobryum alopecurum, Hoockeria lucens y Plagiomnium undulatum, 06-VIII-2016, X. Ignacio González-Martínez, Carlos Boullón Agrelo \& Antía Queiruga del Alisal (SANT 72774).

Con el objetivo de seguir completando los mapas distribución de Cystopteris diaphana elaborados por Quintanilla \& Amigo [1999, sub. C. viridula (Desv.) Desv.] para Galicia y por Moreno 


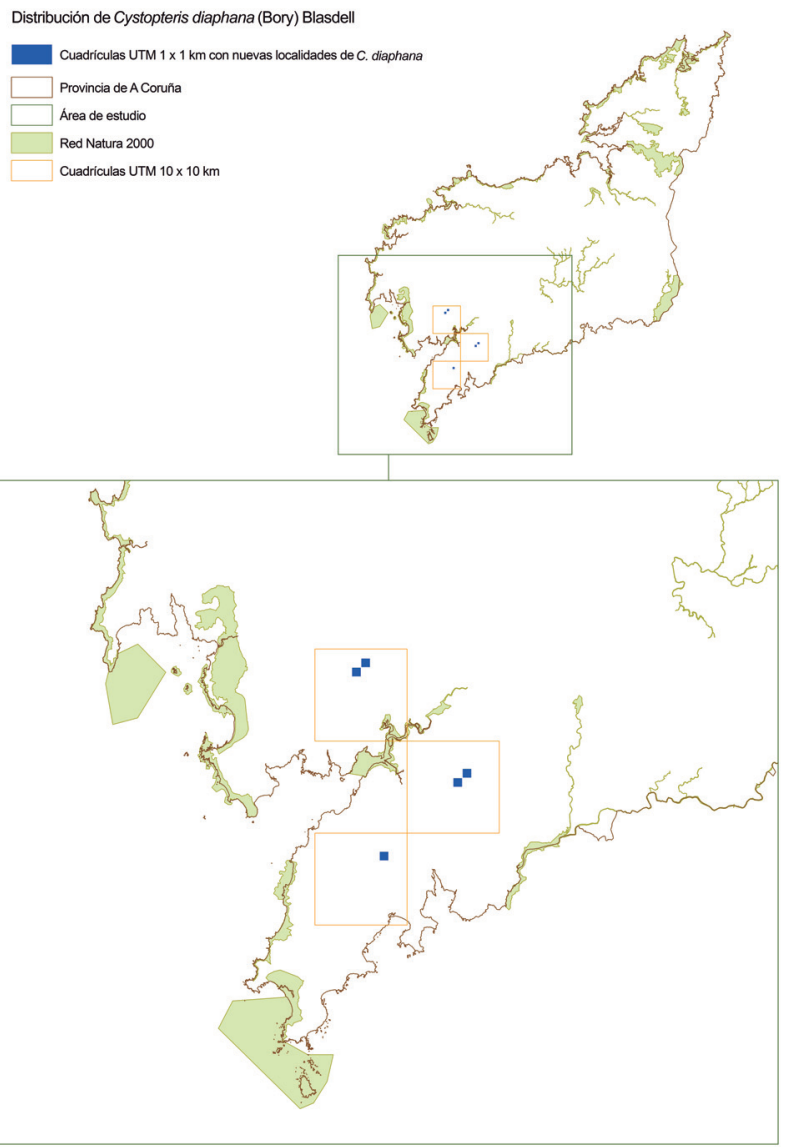

Figura 3. Nuevas localidades de Cystopteris diaphana en el SW de la provincia de A Coruña (Galicia, NW península ibérica). Figure 3. New localities of Cystopteris diaphana in the SW of A Coruña province (Galicia, NW Iberian Peninsula).

Saiz et al. [2015 sub C. viridula (Desv.) Desv.] para el ámbito de la península ibérica e Islas Baleares, presentamos los presentes testimonios correspondientes a varias localidades del SO de la provincia de A Coruña (Fig. 3), ninguna de las cuales se encuentra incluida dentro de las cuadrículas de $10 \times 10 \mathrm{~km}$ proporcionadas por dichos autores.

Para la determinación de los materiales hemos seguido el criterio taxonómico comúnmente utilizado para este género, basado en la ornamentación del perisporio (Jermy \& Harper, 1971; Prada, 1986; Aizpuru et al. 1999; Quintanilla \& Amigo, 1999). Para ello, hemos utilizado microscopía óptica a 600 aumentos. Así mismo, hemos cotejado los resultados de la observación microscópica de las esporas con el patrón de nerviación de la lámina, en base a lo expuesto por Blasdell (1963) y, más recientemente, por Murphy \& Rumsey (2005), encontrando una correlación positiva. Estos autores sugieren que además de los caracteres microscópicos, por lo demás esenciales para una correcta determinación en el género Cystopteris, también resulta de interés tener en cuenta la terminación de los nervios en los ápices de los dientes de las pínnulas, carácter este último de especial utilidad en el campo. De esta manera, $C$. diaphana (Fig. 4) presenta la mayoría de "venas" finalizando en las escotaduras del margen de las pínnulas (Fig. 5), mientras que en el afín $C$. fragilis (L.) Bernh. suelen terminar, en su mayoría, en el

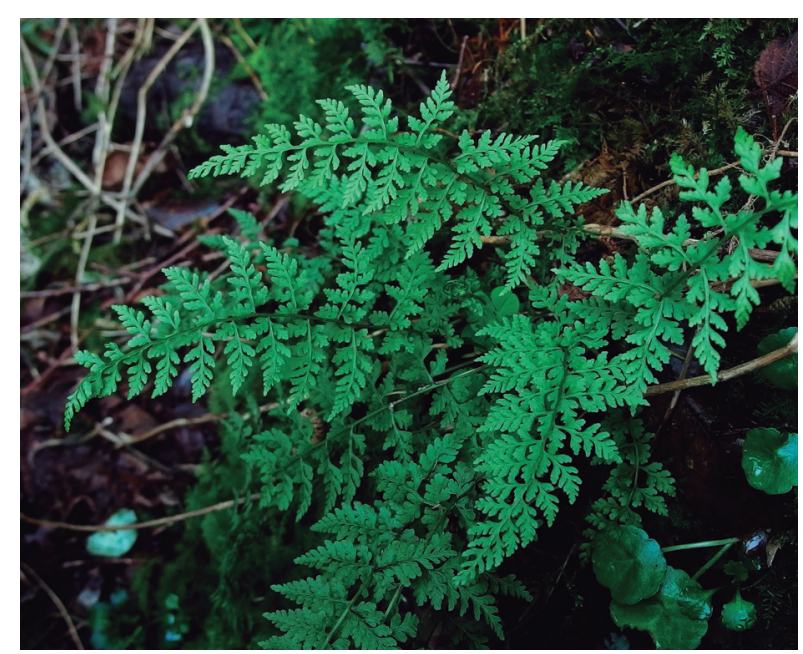

Figura 4. Frondes de Cystopteris diaphana en el campo. Vilacova (Comparada, Lousame, A Coruña); 21-II-2016. Figure 4. Fronds of Cystopteris diaphana in the field. Vilacova (Comparada, Lousame, A Coruña); 21-II-2016.

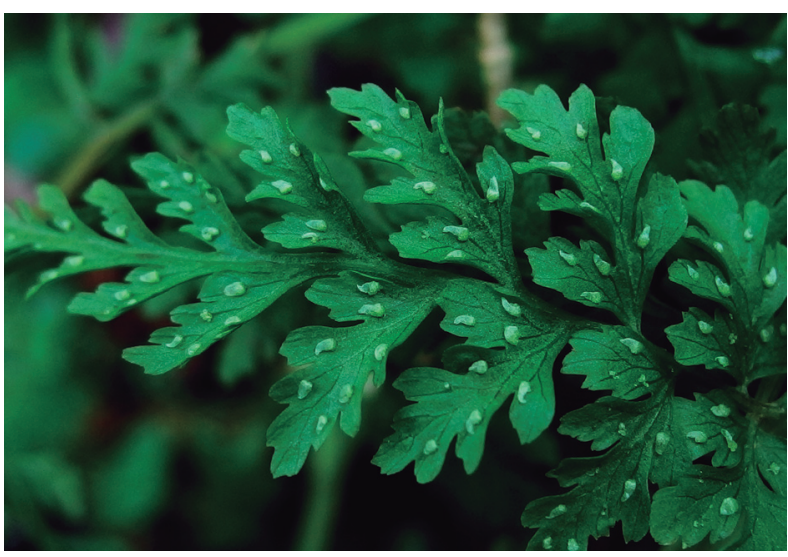

Figura 5. Indusios cuculados característicos del género Cystopteris. Obsérvese que la mayoría de venas terminan en las escotaduras de los lóbulos. Vilacova (Comparada, Lousame, A Coruña); 21-II-2016. Figure 5. Tipical hood-shaped indusium in the genus Cystopteris. Most of veins ending in notches between teeth apices. Vilacova (Comparada, Lousame, A Coruña); 21-II-2016. 
ápice de los dientes de las pínnulas. No obstante, este último carácter taxonómico es puesto en entredicho por Quintanilla \& Amigo (1999).

En cuanto a su ecología, este helecho muestra claras apetencias por los ambientes más oceánicos, y su presencia en Galicia se encuentra ligada sobre todo a enclaves abrigados próximos al litoral, especialmente a fondos de barrancos (Loidi et al. 1997; Quintanilla \& Amigo, 1999). En nuestro caso, siempre lo hemos encontrado ocupando fondos de valle y en la proximidad de cursos de agua, asociado a un bosque litoral atlántico más o menos bien conservado, en ambiente saturado de humedad y en sombra permanente. Por otro lado, y en relación a la sinecología de la especie, Loidi et al. 1997) hablan de una "Comunidad de Cystopteris viridula" asociada a este tipo de ambientes hiperoceánicos, descrita por vez primera como tal para el territorio asturiano (Fernández Prieto et al. 1984), y en la que además de este pteridófito participarían otras plantas ligadas a medios húmedos, como por ejemplo Chrysosplenium oppositifolium L., Cardamine flexuosa With., especies del género Saxifraga L. (en nuestro caso representado por $S$. spathularis Brot.) y Oxalis acetosella L., así como numerosos briófitos. Fitocenosis similares las hemos encontrado en localidades como Comparada (Lousame, Vilacoba; SANT 72621) y a orillas del río San Paio (San Lourenzo de Matasueiros, Outes; SANT 72622).

Por último, y en lo que a su abundancia se refiere, en las vaguadas prospectadas ha resultado una especie de presencia muy puntual y siempre en muy bajo número, por lo que interpretamos se trata de un helecho raro ya no solo en el área prospectada, sino en buena parte de la fachada atlántica gallega, algo que por otra parte sugiere el mapa de distribución de Quintanilla \& Amigo (op. cit.). No obstante, se trata de un género mal conocido en Galicia, debido, entre otros motivos, a determinaciones erróneas, y no solo a nivel específico, sino también genérico. En este sentido, las especies del género Cystopteris han sido frecuentemente confundidas con ejemplares de pequeño porte de Athyrium filix-femina (L.) Roth (Quintanilla \& Amigo, op. cit.). Abundando en lo antedicho, en el Atlas de pteridófitos recientemente elaborado por Moreno Saiz et al. (op. cit.) aparece indicada la presencia de $C$. diaphana (sub $C$. viridula) para el archipiélago de Sálvora (A Coruña, Parque Nacional Marítimo-Terrestre de las Islas Atlánticas de Galicia), quizás debido a su inclusión en el catálogo de flora vascular realizado para dicho complejo insular por Bernárdez Villegas et al. (2012). No obstante, y tras consultar dicha Flora, observamos que la imagen que ilustra la ficha de C. diaphana no parecía corresponderse con esta especie, sino más bien con frondes poco desarrolladas del helecho Asplenium obovatum Viv. subsp. billotii (F.W. Schultz) Kerguélen (=Asplenium billotii F.W. Schultz). Por este motivo, en julio de 2017, hemos visitado el único punto del archipiélago donde supuestamente se encontraba C. diaphana, concretamente en la "Fonte de Santa Catalina" de la Isla de Sálvora, confirmando finalmente nuestra impresión inicial. Por tanto, consideramos que $C$. diaphana debería ser excluido del catálogo florístico del archipiélago de Sálvora.

\section{Agradecimientos}

Al siempre amable y diligente personal del Herbario SANT de la Universidade de Santiago de Compostela, Davide San-León, Ramiro Iglesias y Javier Amigo. A Gabriel Lojo Pose por la confección del mapa con las nuevas localidades de Cystopteris diaphana, y a Antía Queiruga del Alisal, por su compañía en muchas de las jornadas de campo realizadas. Por último, agradecer la labor de dos revisores anónimos cuyas aportaciones han contribuido de forma notable a la mejora del manuscrito inicial.

\section{Referencias}

Aizpuru, I., Aseginolaza, C., Uribe-Echebarría, P.M., Urrutia, P. \& Zorrakin, I. (1999). Claves ilustradas de la flora del País Vasco y territorios limítrofes. Servicio Central de Publicaciones del Gobierno Vasco, VitoriaGasteiz. $831 \mathrm{pp}$.

Bernárdez Villegas, J.G., Blanco-Dios, J.B., Mouriño Lourido, J. \& Rigueiro Rodríguez, J.A. (2012). Flora y vegetación del Archipiélago de Sálvora (Parque Nacional Marítimo-Terrestre de las Islas Atlánticas de Galicia). Organismo Autónomo Parques Nacionales, Ministerio de Medio Ambiente y Medio Rural y Marino, Madrid. $464 \mathrm{pp}$.

Blasdell, R.F. (1963). A monographic study of the fern genus Cystopteris. Mem. Torrey Bot. Club 21: 1-102.

Fernández Prieto, J.A., Fernández Ordóñez, M.C. \& Collado, M.A. (1984). Datos sobre la distribución y el comportamiento ecológico de Cystopteris viridula en Asturias. Bol. Cien. Nat. I.D.E.A 33: 39-48.

Fraser-Jenkins, C.R. (1982). Dryopteris in Spain, Portugal and Macaronesia. Bol. Soc. Brot. Sér. 2, 55: 175-336.

Fraser-Jenkins, C.R. \& Reichstein, T. (1984). Dryopteris. In: K. U. Kramer (ed.), Pteridophyta in G. Hegi, Illustrierte Flora von Mitteleuropa, ed. 3, 1(1): 136169. Berlin, Hamburg.

Gibby, M. (1983). The Dryopteris dilatata complex in Macaronesia and the Iberian Peninsula. Acta. Bot. Malac. 8: 59-72.

Ivanova, D. (2004). Dryopteris affinis subsp. borreri (Pteridophyta: Dryopteridaceae) in the Bulgarian flora. 
Fl. Medit. 14: 201-218.

Jermy, A.C. \& Harper, L. (1971). Spore morphology of the Cystopteris fragilis complex. Brit. Fern Gaz 10: 211213.

Loidi, J., Biurrun, I. \& Herrera, M. (1997). La vegetación del centro-septentrional de España. Itinera Geobot. 9: 161-618.

Murphy, R.J. \& Rumsey, F.J. (2005). Cystopteris diaphana (Bory) Blasdell (Woodsiaceae) - an overlooked native new to the British Isles? Watsonia 25: 255-263.

Pérez Carro, F.J. \& Fernández Areces, M.C. (2007). Dryopteris remota en Cantabria y acerca de un nuevo híbrido: Dryopteris × alejandrei. Flora Montib. 37: 2938.

Pérez Carro, F.J. \& Fernández Areces, M.C. (2013). Dryopteris $\times$ orexpansa, un nuevo híbrido y $D$. $\times$ deweveri en Cantabria. Flora Montib. 54: 150-161.

Pérez Carro, F.J. \& Fernández Areces, M.C. (2016). Dos nuevos híbridos de Dryopteris guanchica: $D$. $\times$ cantabrica y $D . \times$ ronald-vianensis. Flora Montib. 63 : 64-81.

Prada, C. (1986). Cystopteris Bernh. En: S. Castroviejo, M. Laínz, G. López González, P. Montserrat, F. Muñoz Garmendia, J. Paiva \& L. Villar (eds.), Flora iberica I. Lycopodiaceae-Papaveraceae: 115-121. Real Jardín
Botánico, C.S.I.C., Madrid.

Prelli, R. (2015). Guide des Fougères et plantes alliées. Ed. Belin, France. 224 pp.

Quintanilla, L.G. \& Amigo, J. (1999). Distribución del género Cystopteris Bernh. (Athyriaceae, Pteridophyta) en Galicia (NO de la Península Ibérica). Nova Acta Ci. Compostelana (Biol.) 9: 117-124.

Quintanilla, L.G., Viane, R.L.L. \& Van Den Heede, C.J. (2006). Dryopteris $\times$ gomerica, un retrocruzamiento relativamente frecuente en el noroeste de España. Lazaroa 27: 133-135.

Moreno Saiz, J.C., Pataro, L. \& Pajarón Sotomayor, S. (2015). Atlas de de los pteridófitos de la Península Ibérica e Islas Baleares. Acta. Bot. Malac. 40: 5-55.

Salvo, Á.E. \& Arrabal, M.I. (1986). Dryopteris Adans. En: S. Castroviejo, M. Laínz, G. López González, P. Montserrat, F. Muñoz Garmendia, J. Paiva \& L. Villar (eds.), Flora iberica I. Lycopodiaceae-Papaveraceae: 128-143. Real Jardín Botánico, CSIC, Madrid.

Wagner, W.H. jr. (1971). Evolution of Dryopteris in relation to the Appalachians. In: P. C. Holt (ed.), The distributional history of the biota of the southern Appalachians. Res. Div. Monogr. 2: 147-192. Virginia Polytechnic Institute and State University, Blacksburg, Virginia, USA. 